OP005/\#352

SAFETY OF VAGINAL HYSTERECTOMY FOR CERVICAL CANCER: A MULTICENTER COHORT STUDY ON BEHALF OF THE 4C (CANADIAN CERVICAL CANCER COLLABORATIVE) WORKING GROUP

${ }^{1} \mathrm{~N}$ Cockburn, ${ }^{1} \mathrm{G}$ Pond, ${ }^{1} \mathrm{~L}$ Elit, ${ }^{2} \mathrm{D}$ Vicus, ${ }^{2} \mathrm{~S}$ Piedimonte, ${ }^{3} \mathrm{G}$ Nelson, ${ }^{3} \mathrm{C}$ Aubrey, ${ }^{4} \mathrm{M}$ Plante, ${ }^{4} \mathrm{~L}-\mathrm{A}$ Teo-Fortin, ${ }^{5} \mathrm{~S}$ Lau, ${ }^{6} \mathrm{~J}$ Kwon, ${ }^{7} \mathrm{~A}$ Altman, ${ }^{7} \mathrm{~N}-\mathrm{B}$ Saunders, ${ }^{8} \mathrm{~K}$ Willows, ${ }^{8} \mathrm{~N}$ Sadeq, ${ }^{9} \mathrm{~T}$ Feigenberg, ${ }^{10} \mathrm{~J}$ Sabourin, ${ }^{11} \mathrm{~V}$ Samouëlian, ${ }^{1} \mathrm{~L}$ Helpman*. ${ }^{1}$ McMaster University, Juravinski Cancer Center, Hamilton Health Sciences, Gynecologic Oncology, Hamilton, Canada; ${ }^{2}$ University of Toronto, Gynecologic Oncology, Toronto, Canada; ${ }^{3}$ University of Calgary, Department of Oncology, Division of Gynecologic Oncology, Calgary, Canada; ${ }^{4}$ Hotel Dieu de Quebec, Laval University, Gynecologic Oncology, Quebec City, Canada; ${ }^{5}$ McGill University, Jewish General Hospital, Gynecology Oncology, Montreal, Canada; ${ }^{6}$ Vancouver General Hospital, Gynecologic Oncolgoy, Vancouver, Canada; ' University of Manitoba, Gynecologic Oncology, Winnipeg, Canada; ${ }^{8}$ Dalhousie University, Gynecologic Oncology, Halifax, Canada; ${ }^{9}$ Trillium Health Partners/University of Toronto, Gynecologic Oncology, Mississauga, Canada; ${ }^{10}$ Alberta Health Service, Gynecologic Oncology, Edmonton, Canada; "Gynecologic Oncology Service, CHUM, Université de Montréal, Department of Obstetrics and Gynecology, Montreal, Canada

\subsection{6/ijgc-2021-IGCS.22}

Objectives Inferior outcomes of minimally invasive surgery (MIS) in cervical cancer may be attributable to exposure of peritoneum to tumor at colpotomy. Vaginal surgery may minimize surgical morbidity while avoiding dissemination. We sought to compare cervical cancer outcomes by surgical approach.

Methods A retrospective cohort study of cervical cancer patients in ten Canadian centers between 2007-2017. Patients with FIGO 2018 stage IA1, LVI+, and stages IA2-IIIC tumors $<4 \mathrm{~cm}$ were included. Patients undergoing MIS, abdominal (AH) and vaginal or laparoscopy-assisted vaginal hysterectomy $(\mathrm{CLVH})$ were compared. PFS and OS were assessed using the product-limit method, and Cox regression was performed to evaluate association of surgery with outcomes.

Results 1066 patients met inclusion criteria (518 MIS, $436 \mathrm{AH}$ and $110 \mathrm{CLVH}$ ). Radical hysterectomy was performed in $80 \%$ (CLVH), 96.9\% (MIS) and 89.8\% (AH) of cases. CLVH cases included more adeno/adenosquamous cancers $(70.9 \%$ vs. $38.3 \%$ (MIS) and 50\%(AH), p<0.001), more microinvasive disease $(30.9 \%$ vs $21.4 \%$ (MIS) and $15.8 \%(\mathrm{AH}), \mathrm{p}=0.005)$, smaller tumors ( $8 \mathrm{~mm}$ vs. $13 \mathrm{~mm}(\mathrm{MIS}), 15 \mathrm{~mm}(\mathrm{AH}), \mathrm{p}=0.006)$, fewer LVI+ (20.9\% vs. $39 \%(\mathrm{MIS}), 35.9 \%(\mathrm{AH}), \mathrm{p}=0.001)$ and similar rates of lymphatic spread (11.1\% vs $11.1 \%(\mathrm{MIS}), 9.7 \%(\mathrm{AH}))$. CLVH was associated with fewer intraoperative $(5.6 \%$ vs $5.6 \%$ (MIS), $10.1 \%(\mathrm{AH}), \mathrm{p}=0.023)$ and postoperative $(11.8 \%$ vs $18.9 \%(\mathrm{MIS}), 24.5 \%(\mathrm{AH}), \mathrm{p}=0.006)$ complications and readmissions $(4.6 \%$ vs. $11.9 \%(\mathrm{MIS}), 13.9 \%(\mathrm{AH}), \mathrm{p}=0.028) . \mathrm{CLVH}$ was further associated with a lower risk of recurrence, even when adjusted for age and stage $(\mathrm{HR}=2.6,95 \%$ CI $1.04-6.51$ $(\mathrm{AH})$ and $\mathrm{HR}=3.07$, 95\% CI 1.23-7.64 (MIS)).

Conclusions CLVH for cervical cancer is associated with excellent perioperative outcomes. Oncological outcomes appear promising and warrant prospective exploration.

\section{OP006/\#489 SURVIVAL IMPACT OF ONTOGENETIC SURGERY FOR NEWLY DIAGNOSED CERVICAL CANCER}

${ }^{1} \mathrm{U}$ Kim*, ${ }^{2} \mathrm{D}$ Kim, ${ }^{3} \mathrm{SJ}$ Park, ${ }^{4} \mathrm{M}$ Lee, ${ }^{4} \mathrm{HH}$ Chung, ${ }^{3}$ J-W Kim, ${ }^{4} \mathrm{NH}$ Park, ${ }^{4}$ Y-S Song, ${ }^{5} \mathrm{HS}$ Kim. ${ }^{1}$ Seoul National University Hospital, Department of Obsteretics and Gynecology, Seoul, Korea, Republic of; ${ }^{2}$ Seoul National University Hospital, Gynecology, Seoul, Korea, Republic of; ${ }^{3}$ Seoul National University College of Medicine, Obstetrics and Gynecology, Seoul, Korea, Republic of; ${ }^{4}$ Seoul National University College of Medicine, Department of Obstetrics and Gynecology, Seoul, Korea, Republic of, ${ }^{5}$ Seoul National University Hospital, Department of Obstetrics and Gynecology, Seoul, Korea, Republic of

10.1136/ijgc-2021-IGCS.23
Objectives To evaluate the survival impact of ontogenetic surgery for stage IB1-IVB cervical cancer.

Methods We prospectively enrolled patients with stage IB1-IVB cervical cancer (NCT02986568) for patients treated with total mesometrial resection (TMMR) or laterally extended endopelvic resection (LEER) from 2016 to 2020, who received adjuvant chemotherapy if resection margin was positive or positive pelvic lymph nodes $\geq 2$ or positive para-aortic lymph node metastasis. For historical comparison, a retrospective cohort of patients who underwent standard treatment was gathered from 2010 to 2020. Clinico-pathologic characteristics, progressionfree survival (PFS), and overall survival (OS) were compared between the prospective and retrospective cohorts.

Results A total of 46 patients underwent TMMR or LEER in the prospective cohort and 207 patients received standard treatment in the retrospective cohort. Clinico-pathologic characteristics were equally balanced in both cohorts. In terms of survival analysis, ontogenetic surgery showed worse PFS (mean, 53.08 vs 88.3 mons, $\mathrm{p}=0.003$ ) and no differences in OS. In subgroup analysis, stage IB1-IIA2 patients did not show differences in survival, whereas stage IIB-IVB patients showed worse PFS (mean, 30.9 vs. 40.3 mons, $p=0.015$ ) and no difference in OS. In multivariate analysis, ontogenetic surgery was associated with an increase of recurrence (HR, 3.55; 95\% CI, 1.34-9.39)

Conclusions Ontogenetic surgery was associated with increased recurrence in locally advanced cervical cancer despite its similar efficacy to standard treatment in early-stage disease. Thus, we have stopped the recruitment of patients with locally advanced cervical cancer for ontogenetic surgery for considering this harmful effect.

\section{OP007/\#276 PHASE I STUDY OF MIRVETUXIMAB SORAVTANSINE (MIRV) AND RUCAPARIB FOR RECURRENT ENDOMETRIAL, OVARIAN, FALLOPIAN TUBE OR PRIMARY PERITONEAL CANCER}

${ }^{1} \mathrm{~F}$ Backes*, ${ }^{1} \mathrm{~J}$ Fowler, ${ }^{1} \mathrm{~L}$ Copeland, ${ }^{2} \mathrm{~L}$ Wei, ${ }^{1} \mathrm{D}$ O'Malley, ${ }^{1} \mathrm{D}$ Cohn, ${ }^{1} \mathrm{C}$ Cosgrove, ${ }^{3} \mathrm{~J}$ Hays, ${ }^{1} \mathrm{~K}$ Bixel. ${ }^{1}$ The Ohio State University, Obstetrics and Gynecology; Division of Gynecologic Oncology, Columbus, USA; ${ }^{2}$ The Ohio State University, Center For Biostatistics, Columbus, USA; ${ }^{3}$ The Ohio State University, Division of Medical Oncology, Columbus, USA

\subsection{6/ijgc-2021-IGCS.24}

Objectives To estimate the maximally tolerated dose (MTD) and toxicities associated with MIRV and rucaparib.

Methods Patients had to be folate receptor $\alpha$ (FR $\alpha)$ positive by IHC ( $\geq 25 \%$ of tumor staining at $\geq 2+$ intensity). Using a $3+3$ design patients received MIRV (4-6 mg/kg IV every 3 weeks) and rucaparib PO BID (400-600 mg) depending on the dose level.

Results $>100$ patients were screened for FR $\alpha$ expression; 21 have been enrolle, 16 with ovarian and 5 with endometrial cancer. Median age was 64.5, with 3 (range 1-9) prior lines of treatment. 6 patients completed DL2 (5/500), however, 2 DLTs (grade 3 fatigue), let us to establish the RP2D at DL1 (MIRV $5 \mathrm{mg} / \mathrm{kg}$ IV every 3 weeks and rucaparib $400 \mathrm{mg}$ PO BID). Treatment related toxicities (all grades) occurring in $\geq 25 \%$ of patients included fatigue (73\%), nausea (67\%), blurred vision (60\%), anemia (47\%), anorexia (47\%), mucositis (40\%), ALT/AST elevated (40\%), dry eyes (33\%), vomiting (27\%), thrombocytopenia (27\%), weight loss (27\%), leukopenia $(27 \%)$, dysgeusia $(27 \%)$. Grade $\geq 3$ toxicities were fatigue 